\title{
BMJ Open What is the role of a specialist regional mesothelioma multidisciplinary team meeting? A service evaluation of one tertiary referral centre in the UK
}

\author{
Anna C Bibby, ${ }^{1,2}$ Katie Williams, ${ }^{3}$ Sarah Smith, ${ }^{2}$ Nidhi Bhatt, ${ }^{4}$ Nick A Maskell ${ }^{1,2}$
}

To cite: Bibby AC, Williams K, Smith S, et al. What is the role of a specialist regional mesothelioma multidisciplinary team meeting? A service evaluation of one tertiary referral centre in the UK. BMJ Open 2016;6: e012092. doi:10.1136/ bmjopen-2016-012092

- Prepublication history and additional material is available. To view please visit the journal (http://dx.doi.org/ 10.1136/bmjopen-2016012092).

Received 30 March 2016 Revised 11 July 2016 Accepted 13 July 2016

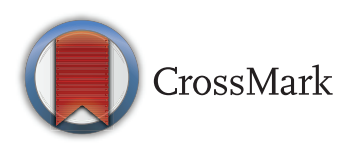

${ }^{1}$ Academic Respiratory Unit, School of Clinical Sciences, University of Bristol, Bristol, UK

${ }^{2}$ Department of Respiratory Medicine, North Bristol NHS Trust, Bristol, UK

${ }^{3}$ Cancer Services, Southmead Hospital, North Bristol NHS

Trust, Bristol, UK

${ }^{4}$ Department of

Histopathology, University

Hospitals Bristol NHS

Foundation Trust, Bristol, UK

Correspondence to

Dr Anna Bibby;

annabibby@doctors.org.uk

\section{ABSTRACT}

Background: Multidisciplinary team meetings are standard care for cancer in the UK and Europe. Professional bodies recommend that mesothelioma cases should be discussed at specialist

multidisciplinary team meetings. However, no evidence exists exploring the role of the specialist mesothelioma multidisciplinary team meeting.

Objectives: To evaluate the clinical activity of 1 specialist mesothelioma multidisciplinary team meeting and to determine how often a definitive diagnosis was made, whether the core requirements of the meeting were met and whether there was any associated benefit or detriment.

Design and setting: A service evaluation using routinely collected data from 1 specialist mesothelioma multidisciplinary team meeting in a tertiary referral hospital in the South-West of England.

Participants: All cases discussed between 1/1/2014 and 31/12/2015.

Outcome measures: The primary outcome measure was whether a definitive diagnosis was made. Secondary outcomes included whether treatment advice was offered, information on clinical trials provided or further investigations suggested. Additional benefits of the multidisciplinary team meeting and time taken from referral to outcome were also collected.

Results: A definitive diagnosis was reached in 171 of 210 cases discussed $(81 \%)$. Mesothelioma was diagnosed in 153/210 (73\%). Treatment advice was provided for 127 of 171 diagnostic cases (74\%) and further investigations suggested for all 35 nondiagnostic cases. $86 / 210$ cases $(41 \%)$ were invited to participate in a trial, of whom $43 / 86(50 \%)$

subsequently enrolled. Additional benefits included the avoidance of postmortem examination if the coroner was satisfied with the multidisciplinary team decision. The overall process from referral to outcome dispatch was $<2$ weeks in $75 \%$ of cases.

Conclusions: This specialist mesothelioma multidisciplinary team meeting was effective at making diagnoses and providing recommendations for further investigations or treatment. The core requirements of a specialist mesothelioma multidisciplinary team meeting were met. The process was timely, with most outcomes returned within 2 weeks of referral.

\section{Strengths and limitations of this study}

- This is the first study to describe the role of the specialist mesothelioma multidisciplinary team meeting.

- The study uses data collected on all cases from one tertiary referral centre over a 2-year period.

- This study will inform the forthcoming British Thoracic Society Mesothelioma Guidelines.

- This is a single-centre study, so results may not be generalisable.

- Dispatch date for multidisciplinary team meeting outcome was missing in $30 \%$ of cases, potentially introducing bias.

\section{INTRODUCTION}

Mesothelioma is an aggressive cancer that predominantly affects the pleural surface. It is incurable, and the only effective chemotherapy regimen offers a median survival benefit of just 2.8 months. ${ }^{1}$ Median survival is less than a year from diagnosis, and consequently care planning needs to be timely as well as effective. ${ }^{2}$

The multidisciplinary team (MDT) is defined by the UK Department of Health as a group of specialists from different healthcare disciplines who meet to discuss a given patient and determine diagnostic and treatment decisions. ${ }^{3}$ MDT meetings have been part of routine cancer care in the UK since the publication of the National Health Service (NHS) Cancer Plan in 2000. ${ }^{4}$ This stipulated that all patients with cancer should be discussed in cancer-specific MDT meetings. ${ }^{4}$ European recommendations followed, leading to the introduction of MDT meetings throughout the continent. ${ }^{5}$

Since their widespread adoption as part of the cancer pathway, MDT meetings have been associated with enhanced patient satisfaction, ${ }^{6}$ increased uptake of chemotherapy ${ }^{7}$ and increased recruitment to clinical 
trials. ${ }^{8}{ }^{9}$ Some studies have reported improved survival outcomes following the implementation of MDT meetings, in particular, in oesophageal, lung, ovarian and breast cancer. ${ }^{10-13}$ However, these observational studies may have been affected by confounding factors, such as concurrent advances in diagnostics or treatment. A systematic review concluded that MDT meetings can influence clinical decision-making, but there is insufficient evidence regarding their effect on outcomes. ${ }^{14}$ Consideration must also be given to the potential pitfalls of MDT meetings, including the resources needed to run the meeting and the potential ramifications of individual clinicians not following MDT advice.

The UK Department of Health's 2007 Mesothelioma Service Framework advised that specialist mesothelioma MDT meetings should be held to discuss cases of diagnostic difficulty where mesothelioma is suspected. ${ }^{15}$ The 2007 British Thoracic Society (BTS) Mesothelioma Statement endorsed this, recommending that challenging cases be referred to a specialist mesothelioma MDT meeting. ${ }^{16}$ Additionally, patients with good performance status should be discussed at mesothelioma MDT meetings to determine appropriate management plans and screen for clinical trial participation. ${ }^{15} 16$

According to the 2007 Mesothelioma Service Framework, ${ }^{15}$ the core requirements of the mesothelioma MDT meeting are:

- To review the pathological and radiological evidence for the diagnosis;

- To make recommendations regarding chemotherapy;

- To encourage the recruitment of appropriate patients to clinical trials.

The BTS is currently writing new guidelines for mesothelioma. Publication is expected in late 2016, and they are likely to encourage the involvement of specialist mesothelioma MDT meetings. However, the worldwide literature currently contains no research either describing the role of the specialist mesothelioma MDT meeting or evaluating its potential benefits or disadvantages.

The aim of this study was to describe the clinical activity of one specialist mesothelioma MDT meeting, and to determine how effective it was in terms of achieving a definitive diagnosis. Secondary aims were to review whether the Mesothelioma Service Framework core requirements were met, and to assess whether the mesothelioma MDT meeting was associated with any additional benefit or detriment. Of specific interest was the time taken for the MDT meeting process, as delays could impact on treatment.

\section{METHODS}

This was a service evaluation of one specialist mesothelioma MDT meeting over a 2-year period.

\section{Setting}

One specialist mesothelioma MDT meeting in the South-West of England, covering one cancer network with a geographical area of 2500 square miles and a population of 2.4 million. The mesothelioma MDT meeting is held once a week, for $30 \mathrm{~min}$ to 1 hour. The MDT meeting is attended by a respiratory physician with a special interest in pleural disease, a thoracic oncologist, a respiratory radiologist and a histopathologist who specialises in pleural disease. In addition, a thoracic surgeon is in attendance at $\sim 20 \%$ of meetings.

\section{Identification of cases}

All cases discussed at the mesothelioma MDT meeting between 1/1/2014 and 31/12/2015 were included. Cases were identified from mesothelioma MDT meeting lists recorded prospectively on the Network Cancer Database. Cases were also identified from a local archive of mesothelioma MDT meeting outcomes recorded at the time of discussion, and a separate prospectively collected mesothelioma database (part of the 'Investigating Pleural Disease Study', UKCRN 8960, REC reference number $08 / \mathrm{H} 0102 / 11)$. A case was defined as a single discussion at the mesothelioma MDT meeting, occurring on one occasion. Patients discussed on more than one occasion were treated as separate cases for each discussion episode.

\section{Data collection}

Data were collected from completed MDT meeting proformas, obtained from electronic archives for all patients. An example of the proforma is shown in online supplementary appendix A and the full data extraction strategy demonstrated in online supplementary appendix B.

The referral section of the proforma was completed by clinicians at the referring hospital and emailed or faxed to the mesothelioma MDT meeting coordinator prior to the meeting. This section contained patient information and outlined the advice requested from the MDT meeting. Patient demographics, data on the referring hospital and any diagnoses already made by the local MDT meeting were obtained from the referral section of the proforma.

The outcome section of the proforma was completed by the respiratory physician in attendance at the mesothelioma MDT meeting, at the time of case discussion. The clinical information discussed, the diagnosis made by the mesothelioma MDT, recommendations for treatment and advice about clinical trials were recorded in this section of the proforma.

Free-text advice was sometimes written in the outcome section of the proforma. If this advice did not relate to the prespecified outcome measures, it was defined as 'additional benefit provided by the MDT meeting' and described narratively. One additional benefit that was revealed during the study period was the potential to avoid a postmortem if a diagnosis of mesothelioma was confirmed by the mesothelioma MDT meeting. Since this was not consistently recorded, and because not all the patients discussed during the study period had died, 
the coroner's office was contacted to enquire how many patients had benefitted from this.

Prior to November 2014, proforma use was not universal. Data for patients discussed prior to this date were extracted from the MDT meeting referral letter and MDT meeting outcome letter, obtained from local hospital records. Following the introduction of the MDT meeting proforma in November 2014, referral letters and outcome letters were rarely used, but where they were available, they were obtained and used for data extraction alongside the proforma.

MDT outcomes for all cases were cross-referenced with summary reports recorded by the MDT coordinator onto the National Cancer Database at the time of discussion at the MDT meeting.

The date of referral to and discussion at the mesothelioma MDT meeting were obtained from the MDT meeting proforma, referral letters and outcome letters. The date of dispatch of MDT meeting outcome was obtained from outcome letters (if the electronic proforma was not used) or from the electronic record of sent emails from the MDT meeting coordinator's email account (for electronic proformas).

Clinical trial participation was assessed using an online research registry of clinical trial participants, managed and maintained by the Research and Development department of the NHS Trust where the MDT meeting was based.

Data extraction was undertaken by ACB and KW, with support from SS and NB between 1/2/2016 and 26/2/ 2016.

\section{Ethics approval}

Ethics approval was not necessary for this service evaluation. Data were collected and stored in accordance with local ethics committee guidance and good clinical practice guidelines.

\section{Outcome measures}

The primary outcome was whether a definitive diagnosis was reached, and what the diagnosis was.

Secondary outcomes were whether a diagnosis represented confirmation of an existing diagnosis, change of existing diagnosis to an alternate one, or formation of a new clinicohistoradiological diagnosis. Clinicohistoradiological diagnoses were defined as cases where test results were non-diagnostic in isolation but, when reviewed in combination, were sufficient to allow the specialist MDT to make a definitive diagnosis.

For non-diagnostic cases, it was noted whether the specialist MDT meeting provided advice on subsequent investigations.

Other secondary outcomes included assessment of whether the 2007 Mesothelioma Service Framework core requirements had been fulfilled, that is, whether radiology and pathology were discussed, whether any treatment recommendations were made and whether cases were identified as eligible for clinical trials. The time taken for the MDT process was evaluated using the date of referral to the MDT meeting, date of discussion at the meeting and date of dispatch of MDT meeting outcome back to the original referrer.

Finally, any additional advice or clinical service that was documented in the free-text section of the MDT meeting outcome proforma was collected, and included under the outcome 'additional benefit'. In order to capture all potential benefits, the definition of this outcome was not prespecified, and all comments were included.

\section{Statistical analysis}

Descriptive statistics were used. Characteristics of cases were tabulated, as were MDT meeting outcomes and diagnoses. Binary outcome measures were reported as percentages. Medians and IQRs were reported for timebased outcome measures. Statistical analysis was undertaken by ACB, with support from NAM, using Stata V.13 (StataCorp LP, Texas, USA).

\section{RESULTS}

The mesothelioma MDT meeting discussed 210 cases during the study period. This represented 194 patients, of whom 179 were discussed once, 14 twice and 1 on three occasions.

\section{Case demographics}

Table 1 shows the characteristics of all cases discussed at the mesothelioma MDT meeting. The majority of cases discussed at the mesothelioma MDT meeting were male $(184 / 210 ; 87.6 \%)$, with a mean age of 74 years (range $50-93$ years). WHO performance status ranged from $0-$ able to carry out all normal activity without restriction, to 5-dead (see online supplementary appendix $\mathrm{C}$ for a full description of WHO performance status). ${ }^{17}$ Information on performance status was missing for 36/ 210 cases $(17 \%)$, but otherwise there were no missing data for case characteristics or referral information.

\section{Primary outcome}

Data were obtained for all cases. Of 210 cases, a definitive diagnosis was made in $171(81 \%), 35(17 \%)$ were non-diagnostic and $4(2 \%)$ had established mesothelioma and were referred to the MDT meeting for advice (table 2).

Of the 171 diagnostic cases, the mesothelioma MDT meeting decision represented confirmation of an existing diagnosis in $127(74 \%)$. In 22 of 171 diagnostic cases $(13 \%)$ the original diagnosis was overturned and an alternate diagnosis made, and in another 22/171 (13\%) a clinicohistoradiological diagnosis was made.

Mesothelioma was diagnosed in 153 of 171 diagnostic cases $(89 \%)$, of which histological subtype was confirmed in 134/153 (88\%). In all 35 non-diagnostic cases, subsequent investigations were advised. In the majority of cases a repeat pleural biopsy $(19 / 35 ; 54 \%)$ was 
Table 1 Summary of cases discussed at the mesothelioma multidisciplinary team (MDT) meeting

\begin{tabular}{lc}
\hline & N (\%) \\
\hline Total MDT meeting discussions & $210(100)$ \\
Total number of patients discussed & 194 \\
Patients discussed on 1 occasion & 179 \\
Patients discussed on 2 occasions & 14 \\
Patients discussed on 3 occasions & 1 \\
Gender & \\
Male & $184(87.6)$ \\
Female & $26(12.4)$ \\
Age (years) & \\
Range & $50-93$ \\
Mean & 74 \\
SD & 7.86 \\
WHO performance status & \\
0 & $45(21.4)$ \\
1 & $77(36.7)$ \\
2 & $26(12.3)$ \\
3 & $22(10.5)$ \\
4 & $1(0.5)$ \\
5 & $3(1.4)$ \\
Not recorded & $36(17.1)$ \\
Referred by: distance & \\
Hospital A 0 miles & $75(35.7)$ \\
Hospital B 13 miles & $41(19.5)$ \\
Hospital C 48 miles & $35(16.7)$ \\
Hospital E 45 miles & $26(12.4)$ \\
Hospital F 35 miles & $12(5.7)$ \\
Hospital G 5 miles & $9(4.3)$ \\
Hospital H 38 miles & $1(0.3)$ \\
Hospital J 123 miles & $1(0.5)$ \\
\hline & $1(0.5)$ \\
\hline
\end{tabular}

advised, with guidance on which technique of obtaining tissue was likely to be most beneficial. In 12/35 (34\%) non-diagnostic cases an interval CT scan was recommended.

\section{Secondary outcomes}

Data on secondary outcomes were available for all cases, except date of dispatch of MDT meeting outcome, which was missing in $61 / 210$ cases (29\%).

Regarding the core requirements as stated in the 2007 Mesothelioma Service Framework, ${ }^{15}$ the mesothelioma MDT meeting reviewed both radiology and pathology in $185 / 210$ cases $(88 \%)$. Radiology alone was reviewed in $17 / 210$ cases $(8 \%)$ and pathology alone in $7 / 210$ cases (3\%). Treatment advice was offered in 127 of 171 diagnostic cases $(74 \%)$ and all four cases referred to the MDT meeting for advice. Advice included palliative chemotherapy, prophylactic/palliative radiotherapy or both in $94 / 127$ cases $(72 \%)$ and active symptom control in 26/127 (20\%). Observation was recommended for 9/ 127 cases $(7 \%)$ with non-malignant diagnoses. Of 210 cases, $86(41 \%)$ were invited to participate in a clinical trial, of whom $43 / 86(50 \%)$ subsequently enrolled.
Table 2 Outcomes of the specialist mesothelioma multidisciplinary team (MDT) meeting

\begin{tabular}{lc}
\hline MDT meeting outcome (all cases) & N (\%) \\
\hline Diagnostic & $171(81)$ \\
Non-diagnostic & $35(17)$ \\
Diagnosis already known, referred for advice & $4(2)$ \\
only & \\
Total & $210(100)$ \\
MDT meeting outcome (diagnostic cases only) & \\
$\quad$ Confirmation of diagnosis made by local & $127(74)$ \\
MDT & \\
Alternative diagnosis made & $22(13)$ \\
$\quad$ Consensus clinicohistoradiological diagnosis & $22(13)$ \\
$\quad$ made & $171(100)$ \\
Total & $90(53)$ \\
Epithelioid mesothelioma & $28(16)$ \\
Sarcomatoid mesothelioma & $12(7)$ \\
Biphasic mesothelioma & $4(2)$ \\
Desmoplastic mesothelioma & $5(3)$ \\
Peritoneal mesothelioma & $14(8)$ \\
Mesothelioma, unspecified & $6(4)$ \\
Other cancer & 1 \\
$\quad$ Lymphoma & 2 \\
Undifferentiated carcinoma & 2 \\
Metastatic adenocarcinoma (lung) & $12(7)$ \\
Metastatic lung cancer & $171(100)$ \\
Metastatic neck cancer & \\
Non-malignant & \\
Total & \\
\hline
\end{tabular}

Referral dates and MDT meeting dates were available for all cases. The median time from referral to discussion at the mesothelioma MDT meeting was 7 days (IQR 6-12 days). In total, $179 / 210$ cases $(85 \%)$ were discussed within 14 days.

The date the MDT meeting outcome was dispatched back to the original referrer was available in $149 / 210$ cases $(71 \%)$. For these cases, the median time from discussion at the MDT meeting to dispatch of outcome was 1 day (IQR 0-1). Outcomes were dispatched within 7 days of MDT meeting discussion in 142/149 cases $(95 \%)$. Median overall time from receipt of referral to dispatch of MDT outcome was 8 days (IQR 7-14). The majority of cases where dispatch date was missing (42/ $61 ; 69 \%)$ were discussed prior to November 2014 and the introduction of electronic MDT meeting proformas. There was no difference in age, sex, performance status, likelihood of achieving a definitive diagnosis or final diagnosis in patients with missing data for this outcome compared with patients without missing data.

In $15 / 210$ cases $(7 \%)$, the mesothelioma MDT meeting offered additional benefit, including a second opinion from an expert mesothelioma pathologist (6/ $210 ; 3 \%)$, compensation advice $(4 / 210 ; 2 \%)$ and a biomarker test not otherwise available $(2 / 210 ; 1 \%)$. Another benefit of the mesothelioma MDT meeting related to postmortems. The coroner covering part of this geographical region agreed that in cases with 
confirmatory pathology, a recent consultant review and previous ratification of the diagnosis at the specialist MDT meeting, postmortem examination was not automatically required. In the 2-year study period, this removed the need for postmortems in 47 patients.

\section{DISCUSSION}

This is the first formal evaluation of a specialist mesothelioma MDT meeting using data collected either prospectively or contemporaneously to clinical practice. The mesothelioma MDT meeting was found to be effective, with high diagnostic rates and advice on subsequent investigations offered in all non-diagnostic cases. The mesothelioma MDT meeting met the core requirements stated by the UK Department of Health's 2007 Mesothelioma Service Framework-to review both histology and radiology, to offer advice on treatment, and to identify potential participants for clinical trials. ${ }^{15}$ In addition to this, the mesothelioma MDT meeting was associated with further benefits, notably removing the need for postmortem examination in cases where the coroner was satisfied with the clinical diagnosis. Finally, the mesothelioma MDT meeting was efficient, with most outcomes sent within 2 weeks of the original referral. This is reassuring, and suggests that referral to the specialist MDT meeting is not associated with significant delays to subsequent treatment or further investigations.

This study had limitations. Although clinical information was collected either prospectively or at the time of the mesothelioma MDT meeting, identification of cases was retrospective. However, use of Network Cancer Database MDT meeting lists ensured comprehensive case identification. Interrogation of alternative data sources did not reveal additional cases, suggesting case identification was robust.

Dispatch dates were missing in one-third of cases, the majority of which were discussed prior to November 2014. The MDT meeting outcome system changed in November 2014 from typed posted letters to emailed proformas. The latter process left a clearer audit trail and hence fewer missing data. However, the electronic system is likely to have resulted in swifter dispatch of outcomes than the previous paper-based system, and therefore bias may have been introduced. Consequently the timings reported in this study should be seen as a reflection of an electronic referral and response system, as for the majority of cases included in the analysis, this was the case.

It is a strength of this study that, apart from dispatch date of MDT meeting outcome and information on patient performance status, there was no missing data. This is likely to be a result of using multiple sources to collect data, and cross-referencing all cases with the National Cancer Database. This also helped remove potential bias from the study, as different data sources were completed by different members of the MDT team (eg, MDT outcome proforma was completed by the respiratory clinician, while data were entered onto the
National Cancer Database by the MDT coordinator). There was no conflict between the data extracted from these different sources.

This study reports data from one specialist mesothelioma MDT meeting. It may not be representative of other mesothelioma MDT meetings, and the generalisability of the results is unknown. While diagnostic success rates should not vary greatly between MDT meetings, other outcomes may differ depending on the frequency of meetings and amount of research activity at each centre. Further studies are needed to determine the efficacy of specialist mesothelioma MDT meetings as a whole.

Similarly, cases of mesothelioma not referred to the regional mesothelioma MDT meeting were not included in this study. Future research could investigate what proportion of mesothelioma cases are referred to the MDT meeting and whether there is a relationship between patient outcomes and discussion at the specialist MDT meeting. Comparing the characteristics and outcomes of patients who are discussed at the mesothelioma MDT meeting with those who are not would be the first step in exploring this area.

This study did not evaluate the role of allied health professionals in the mesothelioma MDT meeting. Future research could focus on the involvement of mesothelioma clinical nurse specialists, referral to palliative care services and whether patient support groups were recommended. A multicentre, randomised trial is currently underway in the UK assessing the impact of early palliative care input on patient-reported, health-related quality of life in mesothelioma. ${ }^{18}$ The outcome of this trial could potentially change practice in mesothelioma, and encourage the involvement of mesothelioma specialist nurses and palliative care physicians in mesothelioma MDT meetings.

This study did not assess survival outcomes or patient satisfaction scores in relation to the mesothelioma MDT meeting. Consequently these results cannot be compared with existing research looking at these outcomes. ${ }^{6}{ }^{10-13}$ This is another area of interest for future research projects.

MDT meetings are part of routine cancer care in the UK, and it would not be possible or ethical to undertake a randomised controlled trial assessing their value. However, in order to maintain and improve standards of care for patients with mesothelioma, specialist MDT meetings should be regularly audited. The development of national standards may be of benefit.

\section{CONCLUSION}

Discussion of mesothelioma cases at this specialist MDT meeting provided the opportunity to review diagnoses, offer advice on investigations and treatment, and encourage recruitment to clinical trials. Evaluation of the service at this single-centre MDT meeting suggests specialist mesothelioma MDT meetings can add value to patient care, without introducing significant time delays. 
Contributors $A C B$ and NAM conceived and designed the study. ACB, KW, SS and NB collected data. ACB undertook the statistical analysis, supported by NAM. ACB wrote the manuscript which was reviewed and approved by all authors. ACB and NAM take complete responsibility for the integrity of the data and the accuracy of the data analysis.

Funding This research received no specific grant from any funding agency in the public, commercial or not-for-profit sectors.

Competing interests All authors have completed the ICMJE uniform disclosure at http://www.icmje.org/coi_disclosure.pdf and declare: ACB has received a small grant from North Bristol NHS Trust Springboard Fund. NAM has received an unrestricted research grant from CareFusion (San Diego, California; USA) and has sat on the advisory board for CareFusion. NAM is Chairman of the British Thoracic Society Mesothelioma Guidelines Committee and is a member of the European Respiratory Society Mesothelioma Guideline Committee.

Provenance and peer review Not commissioned; externally peer reviewed.

Data sharing statement No additional data are available.

Open Access This is an Open Access article distributed in accordance with the Creative Commons Attribution Non Commercial (CC BY-NC 4.0) license, which permits others to distribute, remix, adapt, build upon this work noncommercially, and license their derivative works on different terms, provided the original work is properly cited and the use is non-commercial. See: http:// creativecommons.org/licenses/by-nc/4.0/

\section{REFERENCES}

1. Vogelzang NJ, Rusthoven JJ, Symanowski J, et al. Phase III study of pemetrexed in combination with cisplatin versus cisplatin alone in patients with malignant pleural mesothelioma. J Clin Oncol 2003;21:2636-44.

2. Beckett P, Edwards J, Fennell D, et al. Demographics, management and survival of patients with malignant pleural mesothelioma in The National Lung Cancer Audit in England and Wales. Lung Cancer 2015;88:344-8.

3. Department of Health. Manual for cancer services 2004. London: Department of Health, 2004.

4. UK Department of Health. The NHS cancer plan. London: Department of Health, 2000.
5. Gouveia J, Coleman MP, Haward R, et al. Improving cancer control in the European Union: conclusions from the Lisbon round-table under the Portuguese EU Presidency, 2007. Eur J Cancer 2008;44:1457-62

6. Gabel M, Hilton NE, Nathanson SD. Multidisciplinary breast cancer clinics. Do they work? Cancer 1997;79:2380-4.

7. Boxer MM, Vinod SK, Shafiq J, et al. Do multidisciplinary team meetings make a difference in the management of lung cancer? Cancer 2011;117:5112-20.

8. McNair AG, Choh CT, Metcalfe $\mathrm{C}$, et al. Maximising recruitment into randomised controlled trials: the role of multidisciplinary cancer teams. Eur J Cancer 2008;44:2623-6.

9. Bouvier AM, Bauvin E, Danzon A, et al. Place of multidisciplinary consulting meetings and clinical trials in the management of colorectal cancer in France in 2000. Gastroenterol Clin Biol 2007;31:286-91.

10. Stephens MR, Lewis WG, Brewster AE, et al. Multidisciplinary team management is associated with improved outcomes after surgery for esophageal cancer. Dis Esophagus 2006;19:164-71.

11. Forrest LM, McMillan DC, McArdle CS, et al. An evaluation of the impact of a multidisciplinary team, in a single centre, on treatment and survival in patients with inoperable non-small-cell lung cancer. Br J Cancer 2005;93:977-8.

12. Junor EJ, Hole DJ, Gillis CR. Management of ovarian cancer: referral to a multidisciplinary team matters. $\mathrm{Br} J$ Cancer 1994;70:363-70.

13. Kesson EM, Allardice GM, George WD, et al. Effects of multidisciplinary team working on breast cancer survival: retrospective, comparative, interventional cohort study of 13722 women. BMJ 2012;344:e2718.

14. Croke JM, El-Sayed S. Multidisciplinary management of cancer patients: chasing a shadow or real value? An overview of the literature. Curr Oncol 2012;19:e232-8.

15. Department of Health. Mesothelioma service framework. London: Department of Health Lung Cancer and Mesothelioma Advisory Group, 2007.

16. British Thoracic Society Standards of Care Committee. BTS statement on malignant mesothelioma in the UK, 2007. Thorax 2007;62(Suppl 2):ii1-19.

17. Orr ST, Aisner J. Performance status assessment among oncology patients: a review. Cancer Treat Rep 1986;70:1423-9.

18. Gunatilake S, Brims FJ, Fogg $C$, et al. A multicentre non-blinded randomised controlled trial to assess the impact of regular early specialist symptom control treatment on quality of life in malignant mesothelioma (RESPECT-MESO): study protocol for a randomised controlled trial. Trials 2014;15:367. 